\title{
versants
}

\section{Lettres de Henri Roorda à Jacques Gross}

\author{
Gilles LossERoY \\ Université de Lorraine, laboratoire LIS \\ ORCID: 00oo-0ooI-842I-700I
}

\begin{abstract}
De la correspondance échangée entre J. Gross et H. Roorda de 1894 à I923, seules demeurent les lettres de Roorda. Cet article en propose la synthèse, et la mise en perspective des actions et réflexions des deux correspondants au sein du mouvement anarchiste.
\end{abstract}

Keywords : Henri Roorda, Jacques Gross, anarchie, libertaire, solidarité, lectures, livres, pédagogie.

Jacques Gross (I855-I928) et Henri Roorda (I870-I925) sont deux êtres au mode de vie le plus dissemblable qu'on puisse imaginer. Le premier parcourt l'Europe pour y vanter les bienfaits du tabac dont il tire de confortables ressources, élit domicile à l'hôtel et se restaure aux meilleures tables. Le second, éternel impécunieux, ne quitte la Suisse que pour quelques rares escapades françaises, vivote de son enseignement des mathématiques, qu'il prolonge par la rédaction de manuels scolaires et, sous le pseudonyme de Balthasar, par celle de chroniques drolatiques qu'il livre à la presse quotidienne avec une régularité de métronome. Mais par-delà les contingences, ils adhèrent à un idéal commun : l'anarchie, dont ils sont tous deux ce qu'il est convenu d'appeler des "militants de l'ombre", Gross en finançant et en favorisant la circulation de la presse libertaire, Roorda en se faisant théoricien d'une pédagogie "intégrale".

Les cartes et lettres qu'ils échangent courent de novembre I894 à novembre 1923. Pour Gross, destinataire d'une volumineuse correspondance conservée à l'Institut International d'Histoire Sociale d'Amsterdam (IIHS), Roorda fait figure d'interlocuteur privilégié. Sur les cent dix-sept correspondants qui y sont recensés, Roorda arrive juste après Max Nettlau, qui correspond avec Gross de I893 à 1927. Constance qui s'explique par le fait que Nettlau est l'historien du mouvement anarchiste, et que Gross, par ses voyages et ses nombreuses rencontres, est pour lui un précieux collaborateur. En outre, les deux hommes, qui sont également amis, pourraient se disputer le titre du plus grand collectionneur de revues anarchistes et de la libre pensée. Roorda, lui, a une activité bien plus modeste, et leurs échanges témoignent d'affinités qui excèdent le champ politique. Certes, l'amitié et la solidarité sont consubstantielles à l'idéal anarchiste, mais ici, la sphère privée prend progressivement le pas sur le militantisme, même si elle n'atteint pas le degré d'intimité qui prévaut dans les échanges de Roorda avec une 
autre grande figure militante : Amédée Dunois'. Au fil des lettres envoyées à Gross, le lecteur voit naître le conférencier et s'affirmer la conscience politique du jeune militant de vingt-quatre ans qui s'adresse à un individu de quinze ans son aîné ; deux hommes délaissant rapidement le "vous" pour le "tu", dont Roorda consigne, au fil de soixante-sept missives, une amitié qui se construit et se développe sur une période de près de trente ans. À supposer que le corpus de l'IIHS soit complet, si long que soit cet échange, il n'en demeure pas moins irrégulier : en général deux à quatre lettres dans l'année, avec des interruptions (rien en I90I, I9II, I9I2, I9I5, I9I7, I9I8, I9I9, I922) et des années plus prolixes, comme I909 (dix missives) ou I9I6 (sept missives). Durant cette dernière année, on ne relèvera qu'une seule et très allusive mention à la Première Guerre mondiale. Quant aux quatre lettres de I9I4, elles datent toutes du début de l'année...

Gross, qui est né à Mulhouse, s'engage en politique dès l'adolescence. D'abord de fibre socialiste, il se tourne vers l'anarchie autour de sa vingtième année. Représentant de commerce pour la firme cigarettière Burrus, il met à profit ses voyages pour diffuser les publications socialistes et anarchistes européennes plus ou moins clandestines auxquelles il collabore parfois, de manière anonyme ou sous divers pseudonymes tel Jeanquimarche. Il est lié avec les principales figures de l'anarchisme que sont Nettlau, Galleani, Kropotkin, Malatesta... Relativement fortuné, on le voit souvent ouvrir sa bourse aux militants en difficulté, comme c'est le cas avec Roorda.

Roorda n'est pas devenu anarchiste. Il le fut en quelque sorte de naissance, par transmission des gênes paternels, ceux de l'irascible Sicco Roorda van Eysinga (1825-I887). D’abord ingénieur militaire aux Indes néerlandaises, l'actuelle Indonésie, son caractère impétueux le conduit à démissionner pour se lancer dans la presse locale puis comme administrateur dans une plantation de café sur l'île de Java. Scandalisé par la façon dont le gouvernement batave traite les indigènes, il se rapproche des princes indépendantistes et multiplie les prises de position anticoloniales. Dans son poème-pamphlet Malédiction (Enckell et al. 2009 : 4I), il en appelle à la révolte sanglante contre les Hollandais, ce qui lui vaudra l'admiration de l'écrivain Multatuli. Ses prises de position provoquent son bannissement de la colonie néerlandaise en I864. Après un bref séjour en Hollande puis en Belgique (Henri naît à Bruxelles), Sicco et sa famille s'installent en Suisse en 1872 . La rencontre avec Élisée Reclus, voisin des Roorda à Clarens et qui lui propose de collaborer à sa Géographie universelle, le convertit définitivement aux idées libertaires. Reclus a son couvert chez les Roorda ; le jeune Henri, dont il devient le mentor, aurait été, dit-on, élevé sur ses genoux. Henri entretiendra par la suite une correspondance régulière avec Reclus, qu'il évoque en outre dans ses lettres à Gross.

I La correspondance entre les deux hommes s'étend de 1906 à 1925 (collection particulière). 
De cet ensemble, nous pouvons dégager deux grands axes, que nous ne scindons que pour la commodité de l'étude tant ils sont imbriqués : l'action et la réflexion. Nous en relèverons les éléments les plus saillants, en faisant le choix de laisser de côté les événements familiaux, les conceptions pédagogiques ou le rapport ambivalent de Roorda à l'enseignement, que nous avons eu l'occasion de traiter par ailleurs ${ }^{2}$. L'action se fonde sur la solidarité au sein du mouvement; on la voit se construire en rhizome qui active la fibre amicale autant que politique ; on y voit à l'œuvre les leaders autant que les quidams. La réflexion est celle des doutes et des questionnement personnels des deux correspondants; elle s'enrichit par la mention de leurs lectures, à travers les ouvrages qu'ils se prêtent ou font circuler au sein de leur réseau.

La solidarité est en effet le motif premier de ces échanges, et le premier bénéficiaire en est ici Roorda lui-même qui "tape" régulièrement Gross, fragmente sa dette, repousse les échéances de remboursement avec force humour. La correspondance avec Dunois montre que Roorda ne tend pas son escarcelle vers un seul bienfaiteur... De cet endettement chronique il fera d'ailleurs une des raisons de son suicide, dans un clin d'œil où il prend le contrepied de Socrate :

Quand on ne doit qu'un coq, c'est facile. Moi, je dois mille coqs ; et comme je sais que je n'aurai pas assez d'énergie, pas assez de vertu pour les rendre tous, je vais m'infliger la peine de mort (Roorda 20I7 : 2I).

Gross, qui perçoit très bien la situation de son interlocuteur, lui propose avec tact un arrangement pour ne pas accroître son endettement; il reçoit en retour une lettre significative des difficultés dans lesquelles se débat Roorda (2 décembre 1916) :

Merci d'abord pour les $30 \mathrm{fr}$. que tu m'avances et qui tombent assez bien.

Quant aux 50 fr. que je te dois depuis longtemps, ils n'ont rien d'imaginaire. En dépit de ma tendance au scepticisme, je n'arriverai pas à douter de la réalité de ma dette.

Je n'accepte pas ton arrangement. Car, $-\mathrm{I}^{\circ}$, il n'y a rien dans ma pauvre bibliothèque qui soit digne d'être offert; et puis $-2^{\circ}-$, il serait dangereux pour moi de céder aux suggestions des êtres charitables et généreux dont j'ai le bonheur d'être l'ami.

Étant dans la dèche ces temps-ci, je ne m'[en] acquitterai pas encore $[. . .]^{3}$.

2 Nous n'aborderons pas non plus les débuts littéraires de l'auteur, pour lesquels nous avons déjà sollicité cette correspondance dans plusieurs articles, et auxquels, pour tous ces thèmes, nous renvoyons le lecteur : Enckell et al. (2009:39-59) et Roorda (2013 : 7-19).

3 Pour toutes les lettres citées, nous respectons l'orthographe de l'auteur, ses abréviations et ses particularités (soulignement, etc.). 
Peut-être le travail de comptabilité qu'effectue Roorda pour Gross est-il à mettre aussi au service d'une compensation de ses dettes ? C'est ce qu'on pourrait déduire de deux lettres dans lesquelles Roorda calcule précisément pour Gross des intérêts et échéances de paiement. La première sans doute de 1902, en avril ou mai ; la seconde du 2 mai 1904 :

Au I5 août I906, jour du dernier versement de Ioo frs il sera encore dû I45 frs $83 \ldots-$ au $5 \%$-, ou 175 frs - au $6 \%$ - pour les intérêts.

J'ai calculé dans l'hypothèse des intérêts simples, et non pas des intérêts composés. C'est bien ce que tu entendais, n'est-ce pas?

Les sollicitations de Roorda pour lui-même ne sont pas pour des dépenses d'ordre politique telles que l'achat de matériel de propagande ou des déplacements militants, mais bien pour des difficultés personnelles, comme les frais en prévision de la naissance de sa première fille Béatrice (lettre du 31 juillet 1900) ou les honoraires du médecin accoucheur après la naissance de la seconde, Marguerite (lettre du 24 décembre 1909). De la même manière, Henri interpelle son correspondant sur le cas d'individus qui semblent totalement extérieurs à la sphère libertaire. C'est le cas d'un certain A. J. Vonwiller qu'il présente comme un ami (lettre du 8 février 1903), dont il vante les mérites et égrène le curriculum vitce (lettre du 4 avril 1904) :

Deux mots seulement pour te rappeler que tes amis et connaissances ne pourraient pas trouver un employé plus instruit plus intelligent et plus consciencieux que A. Vonwiller (4, Rue de l'Ecole de Chimie, Genève). Il doit avoir dans les 50 ans. Marié. Pas d'enfants. Il sait le français, l'anglais, l'allemand et l'italien. Il pourrait être secrétaire, s'occuper de la librairie, d'écritures, etc.

Pourrait donner d'excellentes leçons.

Pardonne cette insistance. Elle est désintéressée. Et tu connais tant de monde! Et tu es si gentil ! (!!!!)

Le 2 mai, Roorda remercie Gross, et trois ans plus tard, le 30 avril 1907, il intercède à nouveau pour son ami, insistant très lourdement sur ses difficultés matérielles. De son côté, Gross envoie également des solliciteurs à Roorda, comme le peintre belge Franz Van Montfort (I889-1980), alors dans ses années de formation et qui cherche sans doute à donner à Lausanne quelques cours de dessin et de peinture. Roorda se propose d'évoquer son cas auprès de son ami le galeriste Paul Vallotton (lettre du 22 novembre I9I6).

Le portait de Gross que dessine Roorda à travers ses lettres est celui d'un généreux bienfaiteur qui va parfois jusqu'à devancer les besoins de Roorda et ne réserve pas sa manne à la stricte cause politique. Cependant, c'est principalement au sein de la sphère anarchiste qu'il dispense ses libéralités, 
où il apparaît comme le pivot de la solidarité financière entre les militants, une sorte de banquier clandestin par qui transitent collectes et transferts de fonds. Roorda lui-même y participe dans la mesure de ses faibles possibilités, tantôt financièrement, tantôt matériellement, comme nous l'apprend une lettre non datée de 1898 en réponse à Gross qui retourne une bague envoyée par le couple Roorda :

Si je te dis merci ce n'est pas tant pour la sollicitude dont tu entoures la bague de Lili, cette bague est destinée à être tôt ou tard bazardée pour le bien de quelque fugitif; elle attendra ; l'occasion se présentera [certainem ?]ent.

Il est surtout question dans cette lettre d'une collecte destinée à Nino Samaja qui risque d'être expulsé (sans doute de France, ce qui permet de dater cette lettre de 1898). Roorda envoie à Gross dix-sept francs recueillis auprès d'étudiants et dix de sa part, regrettant de ne pouvoir doubler la mise :

Si par bonheur S[amaja] n'est pas expulsé je tiens à rendre les I7 frs aux deux jeunes gens qui ne sont pas riches. Quant aux Io frs de ton serviteur, tu les enverras à Grave quand tu auras l'occasion de lui envoyer autre chose.

Cette dernière phrase est rayée par Émilie, dite Lili, l'épouse de Henri, qui non seulement témoigne ainsi de son engagement militant auprès de son mari, mais affirme ses choix en précisant en post-scriptum : "C'est moi qui ai biffé cette phrase, parce que il faut que nos io frs soient pour Samaja, car qu'il soit expulsé ou non, il en aura besoin ».

Si le nom de Samaja ${ }^{4}$ témoigne de l'élan de solidarité qui règne au sein de la constellation libertaire, c'est celui de Galleanis qui est le plus emblématique du rôle de Gross en matière d'entraide. En outre, les lettres qui le mentionnent apportent des précisions sur ses lieux de détention.

Condamné en juin I894 à trois ans de prison et deux ans d'assignation à résidence, Galleani est emprisonné à Parme. C'est sans doute là que lui écrit Roorda le 26 novembre I895 avec le projet de lui envoyer des livres par l'intermédiaire de ses parents dont Gross lui a fourni l'adresse. De son côté, Élisée Reclus, à qui Gross avait confié Galleani à son arrivée en Suisse et qui fut quelques mois son secrétaire, se mobilise également. Comme il n'est pas sûr qu'il soit toujours incarcéré à Parme, il s'en remet à Gross pour faire passer un " petit billet » au « brave et vaillant jeune homme » (lettre de Reclus à Gross du 4 octobre I895, IIHS). Le 30 novembre I896, Galleani arrive à son lieu d'assignation : le bagne de Pantelleria, au large de la Sicile. Reclus

4 Nino Samaja (1876-1959), médecin italien qui vécut un temps à Genève et co-fondateur du Réveil avec, entre autres, Luigi Bertoni.

5 Luigi Galleani (I86I-I93I), militant, éditeur et théoricien anarchiste. 
se désole que ni lui ni Gross ne puissent s'y rendre, faute de moyens (lettre de Reclus à Gross ([décembre I896 ?], CIRA ${ }^{6}$ ). Galleani a la ferme intention de s'évader et Reclus échafaude des plans dont il s'ouvre à Gross : «l'argent ne manquera pas [...]. N'est-il pas à conseiller de se diriger sur Malte [...] ? Donnez-moi des détails circonstanciés sur notre ami [...] » (lettre du 8 janvier I897, CIRA). En bon géographe, il rassemble des cartes destinées à décrire à Galleani les côtes qui l'entourent (lettre du 9 février I897, CIRA). Tous deux lèvent des fonds et les envoient à des contacts pas toujours très scrupuleux, en Tunisie notamment. La Crète est également évoquée. Reclus, comme Roorda, envoie aussi des livres à Galleani. Mais invariablement, en cas de difficulté ou de silence prolongé, c'est à Gross que Reclus et Galleani s'en remettent. Galleani finira bien par s'évader, mais de l'île d'Ustica, au nord de Palerme, où il a été transféré début I898, sans doute après un bref passage sur celle de Favignana, comme le relate l'article que lui consacre le Dictionnaire des militants anarchistes ${ }^{7}$. Ce passage par Favignana, absent de la correspondance que nous connaissons, semble attesté par les questions de Roorda qui se plaint (comme Galleani) de la mauvaise écriture de Gross : « tu voudras bien me dire si le nom de son île commence par un $S$ ou par un F » (lettre de Roorda à Gross du I6 février I898). Or, des trois îles fréquentées par Galleani, une seule commence par un F.

Livres et argent circulent entre militants, et Gross est celui qui orchestre les échanges. On le voit également servir d'intermédiaire à son correspondant qui compte sur son éloquent carnet d'adresses pour introduire un ami dans le réseau, en l'occurrence Amédée Dunois, pour qui Gross, qui ne le connaît pas, indique immédiatement des contacts essentiels (réponse à la lettre du 6 juillet 1905$)^{8}$, ou pour donner des conférences. C'est ainsi que le 2 octobre 1909, dans la perspective d'une conférence à Genève, Roorda suggère à Gross de « dire deux mots à l'un de [ses] collègues du Conseil d'État ». Même si l'adage veut qu'"on ne prête qu'aux riches", Gross n'a jamais été Conseiller d'État. Peut-être faut-il prendre ici le terme "collègue" dans un sens maçonnique? À moins qu'il ne s'agisse d'une allusion à Jules-François Perréard (I862-I930), industriel du tabac, et à ce titre peut-être "collègue", qui représenta la Régie française en Suisse en I9I2. Député radical au Grand Conseil genevois (1905-1913), il est Conseiller d'État (1906-19I2) à l'époque de cette lettre. Quelques années plus tard, le 8 novembre I9I6, c'est d'un autre Conseiller d'État que Roorda souhaite s'attirer les bonnes grâces :

6 Centre International de Recherches sur l'Anarchie (Lausanne). Je remercie Marianne Enckell pour sa synthèse sur l'incarcération et l'évasion de Galleani.

7 Disponible en ligne : http://militants-anarchistes.info/spip.php?articler925 [consulté le 25 juin 202I].

8 Brouillon de lettre de Gross (dossier de l'IIHS). 
Si ça ne t'ennuie pas, demande à Quartier-la-Tente si je puis aller faire à Neuchâtel une conférence où je critiquerai avec sévérité, mais avec courtoisie, notre pédagogie.

Édouard Quartier-la-Tente (I855-1925, Genève) est visé par Roorda en raison des affinités politiques qui leur sont communes : cet homme politique est en effet pacifiste, espérantiste et franc-maçon. Nommé directeur des écoles secondaires et supérieures de la ville de Neuchâtel en I896, il est ensuite élu conseiller d'État (radical) du canton de Neuchâtel de I898 à 1922. Il y dirige le Département de l'instruction publique et des cultes. C'est donc à ce titre que Roorda sollicite ce rédacteur d'une nouvelle loi scolaire qui fut admise par le Grand conseil en 1904, mais rejetée par la votation populaire.

Conférences sollicitées, rédaction en cours, analyse des propos tenus ou des retours médiatiques, la question des conférences constitue, avec celle de l'argent, le thème le plus récurrent des lettres écrites par Roorda. Les deux problématiques sont d'ailleurs liées.

"Quand je serai célèbre, je les ferai payer, mes conférences », déclare Roorda le 24 décembre 1909. Quatre ans plus tard, la notoriété serait-elle plus grande, qui fait écrire à Henri : «j'aime autant ne pas faire cette conférence si elle ne doit pas rapporter un centime au père de famille que je suis »? Mais il précise immédiatement : «Il m'arrivera encore de faire des conférences pour rien, pour le plaisir de répandre des idées que j'aime » (lettre du 20 décembre 1913). On sent dans ces lignes la passion de Roorda pour la question pédagogique, mais aussi la nécessité dans laquelle toute sa vie il se sera débattu :

Je voudrais, - pour le plaisir, et pour gagner quelques écus (car je suis toujours dans la dèche) - donner quelques conférences dans la Suisse romande (2 novembre 1916).

«La Pédagogie indiscrète» (lettre du 20 octobre 1909), «Éducation nationale, ou éducation désintéressée » (lettre du [22 février 19I4]), « L'École saitelle ce qu'elle veut?» (lettre du 2 novembre 1916), les titres indiquent sans ambiguïté une critique sévère d'un système éducatif qui, selon Roorda, asservit l'enfant intellectuellement, contraint son corps et l'accable de leçons superflues. Même si les titres ne concordent pas toujours, on peut supposer que ces conférences constituent la matière des articles qu'il publie sous son nom complet de Roorda van Eysinga. On le devine avec la conférence « L'Écolier est-il un prévenu ? ", prononcée à Genève le 27 février I9I4 (lettre du [22 février 19I4]), car à cette question l'auteur a déjà répondu dans le numéro 6 de la revue L'École rénovée (Bruxelles) du 15 septembre 1908 : « L'Écolier est un prévenu ». Pour " L'École et le savoir inutile », la conférence, donnée à Lausanne le 29 janvier puis à Genève le 12 mars I903, précède cette fois 
un article que publie sous le même titre L'École rénovée dans ses livraisons des I5 avril et I5 mai 1908 ( $n^{\circ}$ I et 2). Ces titres témoignent aussi du ton volontiers provocateur qu'adopte Roorda, chez qui Balthasar et sa fibre humoristique semblent toujours en embuscade : « en dépit de l'humour que je laisse percer ici et là, ma conférence est sérieuse » (lettre du 24 décembre 19I3). Si Roorda demande à ce que ses conférences lui rapportent, c'est aussi qu'elles lui coûtent, d'abord en amont, " car une conférence [le] rend nerveux pour 2 ou 3 jours et cela [le] gêne dans [son] travail ", affirme-t-il le 20 décembre I9I3, puis en aval, car « après la légère exaltation que [lui] procure une conférence [...] viennent régulièrement un jour ou deux de dépression » (lettre du 24 décembre I9I3). Cependant, le stress pré-traumatique semble se réduire avec l'expérience : "J'ai donné, il y a 8 jours, une conférence à la maison du Peuple : "L'École et le Savoir inutile". Durant les I5 jours qui ont précédé je n'ai pu penser à autre chose (car c'était la I ${ }^{\text {re }}$ conférence de ma vie) ».

La date, évoquée dans une lettre du 8 février 1903, est d'importance, qui marque l'entrée du pédagogue dans la carrière de conférencier, mais la presse n'a semble-t-il pas consigné l'événement. Si l'on voit Roorda au fil des lettres systématiquement inquiet de la réception de son propos, les comptes rendus qu'en donnera plus tard la Gazette de Lausanne témoignent d'un auditoire enthousiaste, à l'image de ces « quatre cents convertis tressautant de plaisir » à l'écoute de "L'École de demain », cette «satanée conférence » que prépare Roorda lorsqu'il écrit à Gross le [3 janvier 1908] et qui sera donnée le 9 janvier à la Maison du Peuple (édition du io janvier I908), ou de « l'assistance nombreuse » venue entendre «La Bêtise humaine et la pédagogie » à l'invitation du Cercle libéral le 26 janvier 1922 (édition du 29 janvier I908). Las, Roorda reste un grand inquiet qui accepte mal un auditoire trop peu nombreux à son goût et à qui il a l'air de garder rancune, comme au public de Nyon semble-t-il (lettre du 24 décembre I9I3), lors même que la critique est bonne, en tout cas dans La Libre Pensée (lettre du 27 janvier I9I4), dont le titre dit à lui seul la proximité d'idées avec Roorda. Miné par ses inquiétudes, le conférencier ne laisse rien au hasard, pas plus le type de soirée accueillant sa conférence, de gala ou grand-public, que la mixité de l'auditoire :

La conférence que je ferai ne peut convenir, me semble-t-il, qu'à un public mêlé, varié et pas trop restreint. On ne parle pas à 20 messieurs comme on parle à 150 badauds de tout poil et de tous sexes. Ce que je dis là te paraîtra peut-être idiot. Je m'entends fort bien, mais c'est difficile à expliquer. C'est, chez moi, une affaire de nerfs.

Donc, si en dépit de tous les embarras que je te fais, tu veux quand même t'occuper ( 5 minutes) de cette conférence, fais en sorte que les dames soient agréablement mêlées aux messieurs.

Il n'y a pas moyen de l'organiser pour le grand public ? (lettre du 20 décembre 1913). 
Après la conférence vient le temps de l'introspection critique. La lettre du 7 août I9IO, la plus longue de cette correspondance, montre un conférencier mélancolique et impuissant, taraudé par un sentiment de décalage qu'il perçoit également chez son correspondant, conférencier occasionnel lui aussi :

En t'écoutant, je me disais que tu commettais une erreur pareille à celle que je commets souvent. Je m'explique. Toi et moi, nous ne pouvons consacrer que peu de temps à l'expression écrite de nos opinions. Notre métier nous laisse peu de loisirs et il nous fatigue. Aussi, n'est-ce souvent qu'après plusieurs années que nous parvenons à achever l'œuvre, - article de revue ou conférence, - conçue dans un moment d'exaltation. Mais, durant tout ce temps, nous y songeons. Dès le début, certains passages ont déjà leur forme définitive. Nous conservons pour finir certaines phrases, certaines épithètes qui nous ont paru justes en commençant. Or, en quelques années, on change. Et il arrive ainsi que le travail que nous terminons aujourd'hui n'est plus l'expression tout à fait exacte de notre pensée actuelle. [...] j'ai eu l'impression, que les termes que tu employais étaient davantage conformes à des idées que tu as pu émettre naguère en discutant avec des amis ou des adversaires qu'avec tes manières de voir d'à présent. Encore une fois, quelque fermes que soient nos convictions, nous changeons. Et leur expression spontanée à un moment donné n'est pas la même dix ans plus tard.

Très préoccupé moi-même de ne pas laisser travestir mes pensées d'à présent par des formules anciennes restées dans mon vocabulaire, j'ai peutêtre cru voir dans ton cas ce qui se trouve seulement dans le mien.

Cette amertume, cependant, ne dissuade pas Roorda de préparer des conférences, sur un terrain, la pédagogie, dans lequel son engagement libertaire trouve sa pleine mesure. Déterminé à délivrer une parole précise et parfaitement argumentée, Roorda épistolier recherche certains ouvrages, s'exprime sur d'autres qu'il a lus et livre ainsi à la postérité une partie de ses références intellectuelles. Gross n'est pas en reste, qui apparaît là encore en généreux prêteur, bibliothécaire bénévole pourvoyant ses interlocuteurs des volumes qu'ils sollicitent : philosophie, sciences, économie, romans.

Le premier nom qui apparaît ainsi sous la plume de Gross est celui de Burke. Il semble que Roorda, qui est chargé de trouver un de ses opuscules à envoyer à Galleani avec d'autres ouvrages, ne l'ait pas lu. Néanmoins, ce nom est extrêmement représentatif des lectures qui circulent au sein du groupe à l'initiative de Gross, manifestement soucieux de la culture politique de ses amis.

Edmund Burke (1729-I797) est un homme politique et philosophe irlandais à la pensée complexe, à la fois libéral et conservateur. Il publie en 1756 un opuscule anarchiste intitule Vindication of Natural Society: A View of the Miseries and Evils Arising to Mankind. En I79o il s'oppose à la Révolution française dans Réflexions sur la Révolution de France et sur les procédés de certaines 
sociétés à Londres, relatif à cet événement qui eut un grand retentissement en Angleterre et en Europe. Peut-être s'agit-il de l'ouvrage recherché par nos deux correspondants, car Henri se propose de prospecter « chez le direct. de la revue historique vaudoise qui est très ferré sur la Révolut. Française »? Pas plus qu'Henri nous n'avons trouvé trace d'un éditeur « Pott », ni en français, ni en anglais (lettre des I7-I8 juin I895). Auteur représentatif car son nom entre en résonnance avec celui de Mackay, dont Gross a prêté un ouvrage à Roorda, et que ce dernier lui renvoie, bien qu'il n'en ait pas terminé la lecture, faute de temps (lettre du 26 novembre i895). John-Henry Mackay (I864-I933) est un écrivain anarchiste, né en Écosse mais d'expression allemande (il meurt près de Berlin), auteur du roman Anarchistes : mœurs du jour (Die Anarchisten, I89I), qui est peut-être le livre désigné ici. Il est connu également pour sa défense de la cause homosexuelle, et pour avoir publié en I897 Max Stirner, sa vie son œuvre et tiré ainsi de l'oubli l'auteur de L'Unique et sa propriété dont Mackay fait un bréviaire de l'anarchisme individualiste. Nettlau, qui a rencontré Mackay, évoque l'ouvrage dans plusieurs lettres à Gross pour déplorer que l'auteur ne cite pas ses sources : "Mon système a été diamétralement opposé » (lettre non datée, début I898, IISH), précise l'historien de l'anarchisme. Entre Mackay et Burke se dessine, par l'intermédiaire de Stirner, une sorte de filiation d'ordre anarchiste, dans laquelle s'inscrit en filigrane un débat - libéralisme contre anti-libéralisme - qui réapparaît à travers la confrontation avec d'autres lectures, demandées cette fois à Gross par Roorda, en particulier celle de Stuart Mill (lettre du 3 août 1906). On ne s'étonnera pas que Roorda s'intéresse à John Stuart Mill (I806-I873), car le célèbre philosophe et économiste de tendance libérale s'est aussi penché sur l'éducation. Même s'il s'agit pour Mill d'une conversion tardive, il souhaite réconcilier sensibilité et émotions avec la froide pensée logique, ce qui ne peut laisser insensible le professeur de mathématiques. Le " petit traité » que réclame Roorda pour un nouveau prêt est sans doute les Principes d'économie politique (I848). Mill y plaide pour les droits sociaux, déplore l'appât du gain qui déshumanise les travailleurs, et condamne les profits excessifs qui ne profitent qu'aux plus riches et n'entraînent aucun progrès social. À la suite de Mill, Roorda mentionne plusieurs noms qui ont tous à voir avec une pensée de gauche, mais divergent sur la question du libéralisme : «Tu en as peut-être un second à me prêter aussi (de Laveleye, de Gide, de Bastiat ou d'un autre.) Aurais-tu peut-être le Walras ou le Pareto?».

« Je ne t'expliquerai pas aujourd'hui les raisons qui me décident à potasser l'Économie Politique ", annonce d'emblée Roorda à son correspondant (au motif qu'il fait trop chaud), et c'est bien dommage car l'aréopage mentionné, quoiqu'un peu oublié aujourd'hui, est éloquent : Émile de Laveleye (I832-I892) est un penseur socialiste belge, protestant, professeur d'économie politique, et l'auteur entre autres de L'Instruction du peuple (I872). Le sui- 
vant, Frédéric Bastiat (I80I-I850), s'inscrit dans le sillage de J. S. Mill tout en s'en éloignant sur certains points. Économiste et homme politique, c'est un libéral, partisan du libre-échange, qui pourfend le socialisme et le colonialisme. On peut le rapprocher des deux suivants : Léon Walras (I834-I9I0), économiste français, maître de conférences à l'Université de Lausanne, auteur d'une abondante littérature dans laquelle se signale en particulier Éléments d'économie politique pure, ou théorie de la richesse sociale (I874). Il se situe à un point d'équilibre entre socialisme et libéralisme qui empêche de lui assigner une sensibilité politique bien définie. L'Italien Vilfredo Pareto (I848-1923) qui lui succèdera à la chaire d'économie politique de l'Université de Lausanne n'a pas cette ambiguïté. Économiste et sociologue de tendance libérale, il critique le socialisme et le protectionnisme. Les fascistes italiens se réclameront de sa pensée. À l'autre extrémité du spectre politique se situe Charles Gide (I847-I932), économiste et enseignant français, et oncle du célèbre écrivain. Le système coopératif et la structure associative sont au cœur de sa pensée résolument tournée vers l'équité sociale et les valeurs de solidarité. Critique de Walras, il se revendique du socialisme et se rapproche des thèses fouriéristes. Il est l'instigateur d'une troisième voie entre autoritarisme d'État et libéralisme sauvage pour envisager une économie vertueuse qui respecte la liberté individuelle. Il est lui aussi l'auteur de Principes d'économie politique (1883). La variété des sensibilités a des allures de programme universitaire tant le spectre embrassé est large et croise des tendances venues pour la plupart de la gauche, mais évoluant ensuite dans des directions multiples, voire opposées.

Si on peut s'étonner de l'appétit inattendu autant que compulsif de Roorda envers l'économie politique, on en sait davantage sur d'autres lectures : si Gross lui suggère celle de Paul Robin (lettre non datée, peut-être I897), c'est qu'il en a parfaitement saisi l'intérêt pour un jeune enseignant chez qui point l'interrogation pédagogique : «Si c'est un livre sur l'éducation ce serait gentil à toi de me l'envoyer car je suis en plein plongé là-dedans ». La lecture de Robin (I837-I9I2) ne pourra en effet que fortifier la réflexion de Roorda. Enseignant et pédagogue, franc-maçon, Robin fut membre de l'Association internationale des travailleurs (AIT), qu'il quitta en raison de ses affinités libertaires. Il dirigea l'orphelinat de Cempuis (I880-I894) en fervent partisan de l'éducation intégrale dont se réclamera à sa suite Roorda. Lecteur éclectique, Henri affirme également un goût pour les sciences quand, le I2 décembre 1903, il demande à son correspondant « le livre du biologiste Metchnikoff : Études sur la nature de l'homme ("Essai d'une philosophie optimiste") ». Rien d'étonnant à cela, puisqu'« il contient un chapitre intitulé "Qu'est-ce que le progrès ?" or c'est justement le titre de [sa] prochaine conférence ». C'est une des rares conférences dont le titre ne renvoie pas explicitement à l'éducation, et le choix de l'auteur laisse supposer une approche qui 
n'exclut pas une conception médicale du progrès, car le chercheur russe Ilya Ilitch (dit Elie) Metchnikov (I845-I9I6) est zoologiste, bactériologiste et immunologiste, lauréat du prix Nobel de médecine en 1908 avec Paul Ehrlich. Son frère Léon, géographe, fut le secrétaire et l'ami d'Élisée Reclus. Derrière les problématiques d'éducation et de progrès, c'est bien sûr la question sociale qui demeure en toile de fond. Un peu plus tôt, peut-être à l'automne I898, Roorda proposait à Gross de lui rendre un livre de Huret. On peut supposer qu'il s'agit là du journaliste du Figaro Jules Huret (I863-1915), auteur notamment, en I897, de La catastrophe du Bazar de la Charité et Enquête sur la question sociale en Europe. Quelques années plus tard, alors qu'il se trouve en convalescence dans le midi de la France " pour cause de fatigue cérébrale " (lettre du 30 avril 1907), Roorda se rêve en sociologue ou en romancier (lettre du 2 juin 1907). La lecture du Voleur (1898) de Georges Darien', prêté par Gross, a enthousiasmé Henri et Lili (lettre du I7 janvier 1905). Arrière-plan anarchiste de la fin du XIX ${ }^{e}$ siècle, thème de l'argent et du vol comme arme de justice sociale, critique de la bourgeoisie et son système éducatif, réflexions sur la morale, sur la compromission... Le roman a tout pour plaire aux Roorda. Au point de céder à la tentation d'écrire des « romans gais » comme le suggère Gross ? Mais Henri n'a pas - encore - perdu toute ambition, et se berce de la pensée qu'« il serait doux d'avoir, vers la cinquantaine, ou avant, une chaire à l'Université » (lettre du 2 juin 1907).

Cependant l'avenir n'apportera ni chaire ni roman, et pour Roorda la cinquantaine sera courte. Le 6 juillet 1905, Henri sollicitait Gross pour un jeune journaliste inconnu de son correspondant : Amédée Dunois. Vingt ans plus tard, les deux hommes sont devenus des amis, et le 7 novembre 1925 , c'est à Dunois qu'écrit Gross, après avoir reçu une ultime missive de Roorda qui ne figure pas au fond de l'IIHS :

Cher Ami,

Ce matin j'ai reçu une lettre de Roorda - mise à Ioh. du soir à la poste - Il m'annonce qu'il va mourir cette nuit et me fait ses adieux. Navré par cette nouvelle j'ai téléphoné à Lausanne. Il est effectivement mort ce matin à 7 heures. Pauvre Roorda. Je savais que depuis longtemps il broyait du noir, mais je ne m'attendais pas à ce triste dénouement. J'ai pensé te prévenir au cas où il ne t'aurait pas écrit ${ }^{10}$.

De Mme Gross et de moi, à toi, à Madame Dunois et à tes enfants, meilleurs souvenirs.

J. Gross

I, rue du Colombier

9 Le roman, porté à l'écran par Louis Malle en 1967, est accessible en ligne : https://fr.wikisource.org/wiki/Livre:Darien_-_Le_Voleur,_Stock,_I898.djvu [consulté le 25 juin 202I]. 


\section{Bibliographie}

Darien, Georges, Le Voleur, Paris, Stock, I898, disponible sur https://fr.wikisource.org/wiki/Livre:Darien_-_Le_Voleur,_Stock,_I898.djvu [consulté le 25 juin 202I].

Dupuy, Rolf, "GALLEANI, Luigi Giuseppe », Dictionnaire international des militants anarchistes, http://militants anarchistes.info/spip.php?articleı925 [consulté le 25 juin 202I].

Enckell, Marianne et al., Henri Roorda et l'humour zèbre, Lausanne, HumuS/ Association des amis de Henri Roorda, 2009.

Roorda, Henri, Euvres complètes, 2 vol., éd. André Guex, Lausanne, L'Âge d'homme, 1969 (t. I) et 1970 (t. II).

-. Le Rire et les rieurs suivi de Mon suicide, Paris, Mille et Une Nuits, $201 \mathrm{I}$ [1925].

-. Les Saisons indisciplinées, éd. Gilles Losseroy, Doris Jakubec et Carine Corajoud, Paris, Allia, 2013.

-. Ainsi parlait Balthasar, chroniques drolatiques (I920-I92I), Le Thoronet, La Première Heure, 2020. 
